\title{
SiO outflows in high-mass star forming regions: A potential chemical clock? ${ }^{\star}, \star \star$
}

\author{
A. López-Sepulcre ${ }^{1}$, C. M. Walmsley ${ }^{1}$, R. Cesaroni ${ }^{1}$, C. Codella ${ }^{1}$, F. Schuller ${ }^{2}$, L. Bronfman ${ }^{3}$, S. J. Carey ${ }^{4}$, \\ K. M. Menten ${ }^{2}$, S. Molinari ${ }^{5}$, and A. Noriega-Crespo ${ }^{4}$ \\ 1 INAF, Osservatorio Astrofisico di Arcetri, Largo E. Fermi 5, 50125 Firenze, Italy \\ e-mail: sepulcre@arcetri.astro.it \\ 2 Max-Planck-Institut für Radioastronomie, Auf dem Hügel 69, 53121 Bonn, Germany \\ 3 Departamento de Astronomía, Universidad de Chile, Casilla 36-D, Santiago, Chile \\ 4 Spitzer Science Center, California Institute of Technology, Mail Code 220-6, Pasadena, CA 91125, USA \\ 5 INAF - Istituto Fisica Spazio Interplanetario, V. Fosso del Cavaliere 100, 00133 Roma, Italy
}

Received 27 September 2010 / Accepted 19 November 2010

\section{ABSTRACT}

\begin{abstract}
Context. Some theoretical models propose that O-B stars form via accretion, in a similar fashion to low-mass stars. Jet-driven molecular outflows play an important role in this scenario, and their study can help to understand the process of high-mass star formation and the different evolutionary phases involved.

Aims. Observations towards low-mass protostars so far favour an evolutionary picture in which jets are always associated with Class 0 objects while more evolved Class I/II objects show less evidence of powerful jets. The present study aims at checking whether an analogous picture can be found in the high-mass case.

Methods. The IRAM 30-m telescope (Spain) has been used to perform single-pointing SiO(2-1) and (3-2) observations towards a sample of 57 high-mass molecular clumps in different evolutionary stages. Continuum data at different wavelengths, from mid-IR to $1.2 \mathrm{~mm}$, have been gathered to build the spectral energy distributions of all the clumps and estimate their bolometric luminosities.

Results. SiO emission at high velocities, characteristic of molecular jets, is detected in $88 \%$ of our sources, a very high detection rate indicating that there is ongoing star formation activity in most of the sources of our sample. The $\mathrm{SiO}(2-1)$ luminosity drops with $L_{\text {bol }} / M$, which suggests that jet activity declines as time evolves. This represents the first clear evidence of a decrease of $\mathrm{SiO}$ outflow luminosity with time in a homogeneous sample of high-mass molecular clumps in different evolutionary stages. $\mathrm{The} \mathrm{SiO}(3-2)$ to $\mathrm{SiO}(2-1)$ integrated intensity ratio shows only minor changes with evolutionary state.
\end{abstract}

Key words. stars: formation - ISM: clouds - ISM: jets and outflows - ISM: molecules

\section{Introduction}

To understand the formation mechanism of O-B stars, several models which assume high accretion rates (e.g. McKee \& Tan 2003) and/or accretion through massive discs (e.g. Krumholz et al. 2005) have been proposed. So far, a number of deeply embedded massive disc/outflow systems (see Cesaroni et al. 2007) have been found, lending support to such models. However, a detailed picture of the different evolutionary phases involved in the process is still lacking.

Jet-driven molecular outflows play an important role in the accretion scenario (Arce et al. 2007; Ray et al. 2007). An evolutionary sequence for the outflows driven by massive protostars has been proposed by Beuther \& Shepherd (2005), in which an initially well-collimated jet/outflow gradually evolves into a wide-angle wind as the ionising radiation powered by the central massive stellar object becomes more dominant.

\footnotetext{
* Based on observations conducted with the IRAM 30-m telescope near Pico Veleta (Granada, Spain), and the Atacama Pathfinder Experiment (APEX) ESO project: 181.C-0885.. IRAM is supported by INSU/CNRS (France), MPG (Germany), and IGN (Spain). APEX is a collaboration between the Max-Planck-Institut für Radioastronomie, the European Southern Observatory, and the Onsala Space Observatory. $\star \star$ Appendices are only available in electronic form at http: //www. aanda.org
}

López-Sepulcre et al. (2010; LS10 hereafter) studied a sample of high-mass molecular clumps in different evolutionary stages, in the $\mathrm{HCO}^{+}(1-0), \mathrm{HCN}(1-0)$, and $\mathrm{C}^{18} \mathrm{O}(2-1)$ transitions with the IRAM 30-m telescope. The detection of outflows was inferred from the presence of extended non-gaussian wings in the $\mathrm{HCO}^{+}(1-0)$ spectra, together with the spatial distribution of the blue and red lobes. The results show that molecular outflows are present in $75 \%$ of the sources.

Although $\mathrm{HCO}^{+}$can trace molecular outflows, contamination from the infalling envelope makes it difficult to separate the outflow component and determine the collimation degree. Further investigation in a reliable jet tracer is needed. $\mathrm{SiO}$ emission is ideal for this purpose, because its formation is attributed to sputtering or vaporisation of $\mathrm{Si}$ atoms from grains due to fast shocks (Gusdorf et al. 2008; Guillet et al. 2009), and thus suffers minimal contamination from quiescent or infalling envelopes. While in the low-mass case it has been possible to carry out highspatial resolution observations in $\mathrm{SiO}$, revealing well-collimated molecular jets (e.g. Gueth et al. 1999), only a few SiO surveys have been made towards high-mass SFRs which include IR-dark sources, representative of the earliest evolutionary phases (e.g. Motte et al. 2007; Sakai et al. 2010). However, an interesting recent study by Jiménez-Serra et al. (2010) shows that the $\mathrm{SiO}$ emission towards the IR-dark cloud G35.39-0.33 is extended on parsec scale, suggesting widespread low-mass star formation. 
Observations towards low-mass protostars so far point to an evolutionary picture in which jets are always associated with Class 0 objects (e.g. Gueth et al. 1999; Codella et al. 2007), while Class I/II objects show less evidence of powerful jets, consistently with the progressive disappearance of the high-density clump around the young stellar object (YSO). The present study represents a first step to check whether an analogous picture can be found in the high-mass case.

With this in mind, we performed $\mathrm{SiO}(2-1)$ and (3-2) singlepointing observations towards the central positions of the LS10 sample, to search for evidence of emission caused by jets and check for evolutionary trends in the outflow detection rate and jet activity.

\section{The sample}

The sample under study is composed of 57 high-mass, parsecscale molecular clumps. These include the 48 sources studied by LS10, which were selected from the millimetric surveys carried out by Rathborne et al. (2006), Beuther et al. (2002), Faúndez et al. (2004) and Hill et al. (2005); and 9 ultracompact HII (UC HII) regions selected from the maser and/or continuum centimetric surveys performed by Hofner \& Churchwell (1996), Walsh et al. (1998) and Wood \& Churchwell (1989).

The sample has been sub-classified into two groups which supposedly represent two different evolutionary stages of the star formation process. These are, from less to more evolved phases: (i) 20 infrared dark (IR-dark) clumps, i.e., not detected at $8 \mu \mathrm{m}$ with the Midcourse Space eXperient (MSX). Many of these are embedded $24 \mu \mathrm{m}$ sources seen with the Multiband Imaging Photometer of Spitzer (MIPS) on the Spitzer Space Observatory; (ii) 37 infrared loud (IR-loud) clumps, i.e., detected at $8 \mu \mathrm{m}$ by MSX. Within the IR-loud sub-sample, 24 sources are known to contain at least one UC HII region inside a radius of $\sim 20^{\prime \prime}$. In addition, UC HII regions have been detected at $3.6 \mathrm{~cm}$ with the VLA in 4 IR-dark clumps Battersby et al. (2010).

The following criteria are satisfied by the sources in our sample: (i) $\delta>-15^{\circ}$; (ii) $M_{\text {clump }} \gtrsim 100 M_{\odot}$, to prevent contamination by low-mass star forming regions; and (iii) $d<4.5 \mathrm{kpc}$, to limit the spread in distances. The last criterion is satisfied by all but 5 IR-dark clumps which were found a posteriori to have distances greater than $4.5 \mathrm{kpc}$ (LS10).

\section{SiO observations}

The IRAM 30-m telescope near Pico Veleta (Granada, Spain) was used on July 30-31 and August 1-2 2009 to observe our sample in the $\mathrm{SiO}(2-1)$ and $\mathrm{SiO}(3-2)$ lines, with rest frequencies at 86.85 and $130.3 \mathrm{GHz}$, respectively.

For each source, single-pointing observations in wobbler mode were made, with a wobbler throw of $120^{\prime \prime}$ and a total integration time $(\mathrm{ON}+\mathrm{OFF})$ varying between 4 and $60 \mathrm{~min}$, depending on the source. All the lines were covered at high spectral resolution using the autocorrelator VESPA.

The data were reduced using the programs CLASS and GREG of the GILDAS software package developed by the IRAM and the Observatoire de Grenoble. The spectra were smoothed to a resolution of $1.5 \mathrm{~km} \mathrm{~s}^{-1}$ to improve the signal to noise ratio. The typical $1 \sigma \mathrm{rms}$ values of the spectra after smoothing are $0.009 \mathrm{~K}$ for the $\mathrm{SiO}(2-1)$ line, and $0.011 \mathrm{~K}$ for the $\mathrm{SiO}(3-2)$ line.
Table 1. Continuum data.

\begin{tabular}{lccc}
\hline \hline Telescope - Instrument & $\begin{array}{c}\text { Wavelength } \\
(\mu \mathrm{m})\end{array}$ & $\begin{array}{c}\text { Ang. Resol. } \\
\left({ }^{\prime \prime}\right)\end{array}$ & $\begin{array}{c}1 \sigma \mathrm{rms} \\
\left(\mathrm{mJy} \mathrm{beam}^{-1}\right)\end{array}$ \\
\hline MSX - Spirit III & 21.3 & 20 & $\sim 150$ \\
Spitzer - MIPS* & 24 & 6 & $\sim 3$ \\
IRAS & 60 & 60 & $\sim 85$ \\
Spitzer - MIPS* & 70 & 18 & $\sim 40$ \\
IRAS & 100 & 120 & $\sim 300$ \\
APEX - LABOCA & 850 & 18 & $\sim 100$ \\
IRAM 30-m - MAMBO & 1200 & 11 & $\sim 10-15$ \\
SEST 15-m - SIMBA & 1200 & 24 & $\sim 40-150$ \\
\hline
\end{tabular}

Notes. ${ }^{*}$ Spitzer Galactic plane MIPSGAL survey (Carey et al. 2005, 2009). (†) APEX Telescope Large Area Survey of the Galaxy (ATLASGAL) survey (Schuller et al. 2009). ${ }^{(\ddagger)}$ Millimetre surveys cited in Sect. 2.

\section{Continuum observations}

Continuum data at different wavelengths, ranging from midIR to $1.2 \mathrm{~mm}$, have been gathered in order to characterise the emission of the clumps from their spectral energy distributions (SEDs). Table 1 summarises the telescopes, instruments, wavelengths, angular resolution, and sensitivity of the data.

\section{Results and discussion}

\subsection{Spectral energy distributions}

Single-temperature, modified black body functions have been fitted to the SEDs of our sources to estimate their dust temperatures, masses (derived from the dust temperature and the dust continuum emission at $1.2 \mathrm{~mm}$ integrated over the whole clump), and bolometric luminosities (Table A.1). This has been possible for 47 of the observed clumps. For the remaining 10 sources, data from fewer than three of the surveys listed in Table 1 were available and thus the fit was not sufficiently reliable. While the single-temperature modified black body is a simplified model, it is sufficient for our purposes and it characterises the global properties of our dusty clumps.

The luminosities $\left(\sim 10^{3}-10^{6} L_{\odot}\right)$ and masses ( $\sim 50$ to $\sim 1000 M_{\odot}$ ) derived are typical of high-mass SFRs. The IR-dark clumps are on average colder and less luminous (by about an order of magnitude), although their masses are comparable to those of the IR-loud clumps. We note that the bolometric luminosities obtained for the sources using IRAS data represent upper limits to the actual luminosities in that there are often several sources within one IRAS beam. Similarly, upper limit luminosities were derived for 5 IR-dark sources for which only upper limits were available at 24 and/or $70 \mu \mathrm{m}$ emission.

\subsection{SiO outflow detection rate}

$\mathrm{SiO}$ emission has been clearly detected above $3 \sigma$ in $88 \%$ of the sources. We note that, even if the sources with no $\mathrm{SiO}$ detection correspond to spectra with $\sigma$ above the average value, the weakest detection in our sample is well above the $3 \sigma$ level of the noisiest non-detection, and therefore we do not consider our $\mathrm{SiO}$ detection rate to be biased by the sensitivity of the different observed spectra. Out of the 7 non-detections, 6 belong to the IR-loud group, which implies a detection rate of $84 \%$ for the IR-loud sub-sample and $95 \%$ for the IR-dark sub-sample. These high detection rates are similar to the one reported by Motte et al. (2007) for a population of massive cores inside Cygnus X.

The lines display a wide variety of intensities, widths and shapes. Figure B.2 presents the $\mathrm{SiO}(2-1)$ and (3-2) spectra of all 
A. López-Sepulcre et al.: $\mathrm{SiO}$ outflows in high-mass star forming regions: A potential chemical clock?

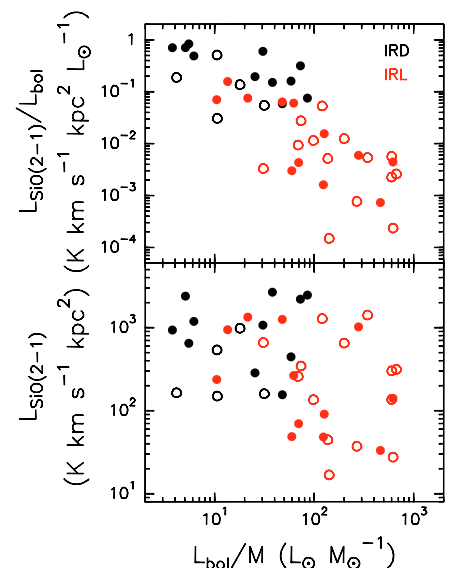

Fig. 1. Top: $\mathrm{SiO}(2-1)$ to bolometric luminosity ratio, $L_{\mathrm{SiO}(2-1)} / L_{\mathrm{bol}}$, against $L_{\text {bol }} / M$. Bottom: $\mathrm{SiO}(2-1)$ luminosity, $L_{\mathrm{SiO}(2-1)}$, against $L_{\text {bol }} / M$. IR-dark and IR-loud clumps are marked as black and red circles, respectively. Open circles depict sources for which the derived luminosity represents an upper limit (see Sect. 5.1).

our detected sources. In all the cases the lines have Full Widths at Zero Power (FWZP) of $\sim 10 \mathrm{~km} \mathrm{~s}^{-1}$ or more. The median FWZP of our sample is $38 \mathrm{~km} \mathrm{~s}^{-1}$ for the $\mathrm{SiO}(2-1)$ line, and $45 \mathrm{~km} \mathrm{~s}^{-1}$ for the $\mathrm{SiO}(3-2)$ line. On average, IR-dark clumps present broader $\mathrm{SiO}(2-1)$ wings than IR-loud clumps, with median values of $49 \mathrm{~km} \mathrm{~s}^{-1}$ for the former and $24 \mathrm{~km} \mathrm{~s}^{-1}$ for the latter. In some cases the $\mathrm{SiO}$ line wings extend up to velocities of about $40-50 \mathrm{~km} \mathrm{~s}^{-1}$ from the systemic velocity of the clump. Such high velocities point to the presence of molecular outflows, likely caused by jets, and therefore active star formation, in most of our sources. Table A.2 lists the measured $\mathrm{SiO}(2-1)$ and (3-2) $F W Z P$ s and velocity-integrated intensities for all the observed sources.

There is good agreement between the detection of $\mathrm{SiO}$ and the presence of $\mathrm{HCO}^{+}(1-0)$ outflows as reported by LS10 (Table A.2): $95 \%$ of the sources with an $\mathrm{HCO}^{+}$outflow present also $\mathrm{SiO}$ emission. There are 6 sources with clear $\mathrm{SiO}$ emission which have no reported $\mathrm{HCO}^{+}$outflow, but they all have $\mathrm{SiO}$ spectra with FWZP lower than about $15 \mathrm{~km} \mathrm{~s}^{-1}$, and $\mathrm{HCO}^{+}(1-$ $0)$ spectra with similar widths. This is compatible with molecular outflows oriented close to the plane of the sky: the narrowest $\mathrm{SiO}$ spectra likely have counterpart $\mathrm{HCO}^{+}(1-0)$ spectra where the blue and red outflow wings are mixed with the low ambient velocities of the line. Our results therefore confirm that $\mathrm{SiO}$ is closely associated with molecular outflows. As an example, Fig. B.1 presents the $\mathrm{SiO}(2-1)$ and (3-2) spectra of two of our targets (one IR-loud and one IR-dark), with an overlay of their corresponding $\mathrm{HCO}^{+}(1-0)$ spectra (LS10).

\subsection{Decrease of SiO outflow activity with time}

It is reasonable to expect that the bolometric luminosity, $L_{\mathrm{bol}}$, of a molecular clump will increase as time evolves and the highmass star gradually switches on, while its mass will decrease due to the effect of molecular outflows, winds, and HII regions (e.g. McKee \& Tan 2002). Therefore, the luminosity to mass ratio, $L_{\mathrm{bol}} / M$, can be considered to be a time estimator which, in addition, gets rid of distance effects. We will then consider $L_{\mathrm{bol}} / M$ to be a measure of time or evolutionary phase, with lower values corresponding to less evolved stages of cluster formation.

From the integrated intensity of the $\mathrm{SiO}(2-1)$ line, the $\mathrm{SiO}(2-1)$ luminosity, $L_{\mathrm{SiO}(2-1)}$, has been calculated. Figure 1 (top) displays a plot of $L_{\mathrm{SiO}(2-1)} / L_{\mathrm{bol}}$ against $L_{\mathrm{bol}} / M$ for the

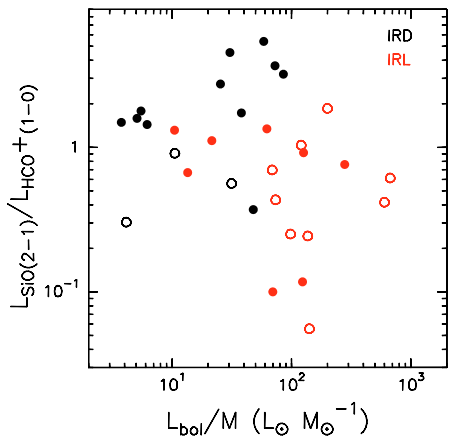

Fig. 2. Ratio of $\mathrm{SiO}(2-1)$ luminosity to $\mathrm{HCO}^{+}(1-0)$ line wing luminosity, $L_{\mathrm{SiO}(2-1)} / L_{\mathrm{HCO}^{+}(1-0)}$, against $L_{\mathrm{bol}} / M$. The same symbols as in Fig. 1 apply here.

47 sources where the SED could be fitted by a modified black body function (see Sect. 4.1). IR-dark and IR-loud clumps are depicted in black and red, respectively. One notes that the two types of object occupy two distinct areas of the plot. This plot shows that the most luminous $\mathrm{SiO}$ outflows are associated with the youngest stages of high-mass star and cluster formation. In the lower panel of Fig. $1, L_{\mathrm{SiO}(2-1)}$ is plotted instead of $L_{\mathrm{SiO}(2-1)} / L_{\text {bol }}$. We note that the results in the upper panel of Fig. 1 have an inherent bias in that the bolometric luminosity appears both in the denominator of the ordinate and in the numerator of the abscissa. This however is not the case in the lower panel, where nonetheless one observes a dearth of points in the lower left hand corner. Although we do not show it here, a similar trend is found when the outflow terminal velocity is plotted against $L_{\text {bol }} / M$.

The trend seen in Fig. 1 may be interpreted as a decrease in the $\mathrm{SiO}$ abundance with time (as suggested by Sakai et al. 2010), a decrease in the jet/outflow mass with time, or a combination of both. In an attempt to discriminate between these possibilities, we present in Fig. 2 a plot of $\mathrm{SiO}(2-1)$ to $\mathrm{HCO}^{+}(1-0)$ luminosity ratio, $L_{\mathrm{SiO}(2-1)} / L_{\mathrm{HCO}^{+}(1-0)}$, against $L_{\text {bol }} / M$. $L_{\mathrm{HCO}^{+}(1-0)}$ corresponds to the emission under the line wings defined by LS10. It can be seen that IR-dark sources lie higher in the plot, while high $L_{\text {bol }} / M$ sources are on average lower and have greater dispersion in $L_{\mathrm{SiO}(2-1)} / L_{\mathrm{HCO}^{+}(1-0)}$. This difference between less evolved and more evolved objects favours the interpretation in which $\mathrm{SiO}$ abundance decreases with time, since otherwise one would expect all the points in Fig. 2 to cluster around a constant value of $L_{\mathrm{SiO}(2-1)} / L_{\mathrm{HCO}^{+}(1-0)}$. Moreover, LS10 reported similar outflow masses among the IR-dark and the IR-loud sub-samples, and therefore there is no significant difference in the outflow mass between more evolved and less evolved clumps. Given that $\mathrm{SiO}$ is thought to be produced in fast shocks which can be effectively generated by fast collimated jets, this result suggests that the earliest phases of star formation are dominated by wellcollimated jets, which gradually de-collimate and lose power. Such a decrease in the $\mathrm{SiO}$ outflow luminosity with time seems analogous to what is found for low-mass SFRs (e.g. Gibb et al. 2004), where the most intense $\mathrm{SiO}$ emission is detected towards the less evolved Class 0 objects. This similarity between the lowmass and the high-mass regime lends support to the disc accretion models as the mechanism to form high-mass stars.

It could be argued that the $\mathrm{SiO}$ emission arising from the high $L_{\mathrm{bol}} / M$ sources comes from low-mass YSOs within the region and not from their high-mass neighbours. However, LS10 reported outflow masses and momentum rates consistent with high-mass stars, independently of the evolutionary phase. Therefore, the correlation seen in Fig. 1 most likely 


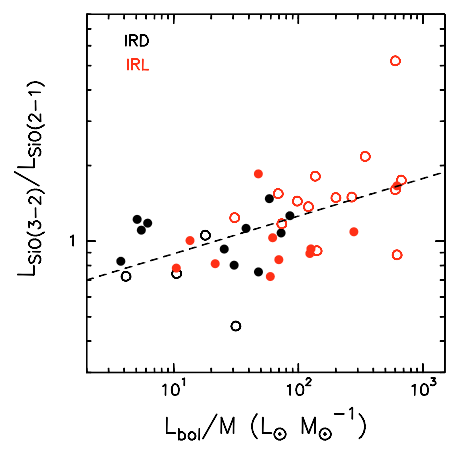

Fig. 3. $\mathrm{SiO}(3-2)$ to $\mathrm{SiO}(2-1)$ luminosity ratio, $L_{\mathrm{SiO}(3-2)} / L_{\mathrm{SiO}(2-1)}$, against $L_{\mathrm{bol}} / M$. The dashed line represents the best fit to the points (see text). The same symbols as in Fig. 1 apply here.

represents an evolutionary line for molecular outflows driven by massive YSOs, compatible with the scenario proposed by Beuther \& Shepherd (2005) in which initially well collimated outflows gradually disappear to make way for a wide-angle wind-dominated outflow. Our result is consistent with the findings of Miettinen et al. (2006), who measured a decrease of the $\mathrm{SiO}$ abundance with core temperature in a sample of $15 \mathrm{UC}$ HII regions and concluded that $\mathrm{SiO}$ outflows are less frequent in more evolved, warmer cores. Given that our sample, which includes IR-dark sources, presumably covers a wider range of evolutionary phases, this represents the first time that clear evidence of a decrease in jet/outflow activity with time is found in a sample of high-mass SFRs.

A final remark concerns the excitation conditions of the jet environment. We checked for a possible dependence of the $\mathrm{SiO}(3-2)$ to $\mathrm{SiO}(2-1)$ intensity ratio on velocity, but a well defined trend has not been discerned. Further, we present in Fig. 3 a plot of $\mathrm{SiO}(3-2)$ to $\mathrm{SiO}(2-1)$ luminosity ratio, $L_{\mathrm{SiO}(3-2)} / L_{\mathrm{SiO}(2-1)}$, as a function of $L_{\mathrm{bol}} / M$. Such a ratio changes by at most a factor 4 over the sampled range of $L_{\mathrm{bol}} / M$. The points follow a weak trend which can be described by the function $L_{\mathrm{SiO}(3-2)} / L_{\mathrm{SiO}(2-1)}=0.63\left(L_{\mathrm{bol}} / M\right)^{0.15}$, with a correlation coefficient of 0.38 , obtained from a least squares fit to all the points. Notice, however, that the the best fit results depend on whether the open circles (representing upper limits to the bolometric luminosity) are included or not, and therefore the correlation found should be confirmed using a more statistically reliable sample. The trend, if real, suggests that the excitation temperature of the post-shock environment increases only marginally with time, which may be explained by a small increase in density with time. To this end, we used the non-LTE excitation code RADEX with an escape probability formalism for the radiative transfer (Van der Tak et al. 2007) coupled with the LAMDA database (Schöier et al. 2005). Assuming that (i) both $\mathrm{SiO}$ lines trace the same region; and (ii) the beam filling factor equals unity, then, for a kinetic temperature between 50 and $150 \mathrm{~K}$, we infer densities between $5 \times 10^{4}$ and $10^{6} \mathrm{~cm}^{-3}$. The total $\mathrm{SiO}$ column densities lie in the range $10^{12}-10^{13} \mathrm{~cm}^{-2}$. A more exhaustive study of the level of excitation of $\mathrm{SiO}$ would require high angular resolution mapping of higher $J$ transitions.

\section{Conclusions}

We have observed $\mathrm{SiO}(2-1)$ and $\mathrm{SiO}(3-2)$ with the IRAM 30$\mathrm{m}$ telescope towards a sample of 57 high-mass molecular clumps in different evolutionary stages, and characterised their IR and (sub)mm emission using continuum data from the archive and a modified grey body function model to fit their SEDs. Highvelocity $\mathrm{SiO}$ emission has been detected in $88 \%$ of our sample at high velocities, implying star formation is actively taking place in most of our targets. A tight inverse correlation is found between $L_{\mathrm{SiO}(2-1)} / L_{\mathrm{bol}}$ and $L_{\mathrm{bol}} / M$, which is interpreted as the presence of an evolutionary line in which jet activity fades as time evolves. This picture is analogous to what is found in the low-mass regime, which suggests that high-mass stars may form generally via disc accretion as low-mass stars. Our result is consistent with the evolutionary picture suggested by Beuther \& Shepherd (2005) for massive molecular outflows. For the first time, evidence of a clear decrease of $\mathrm{SiO}$ outflow luminosity with time has been measured in a sample of high-mass star forming clumps in different evolutionary stages, covering both IR-dark and IR-loud clumps. A relatively constant luminosity ratio of $\mathrm{SiO}(3-2)$ to (2-1) integrated intensity is found in our sample, suggesting that the post-shock density does not vary greatly. We note, however, that high-angular resolution imaging is necessary to confirm our conclusions.

Acknowledgements. A.L.S. acknowledges support from the FP6 Marie-Curie Research Training Network "Constellation: the origin of stellar masses" (MRTN-CT-2006-035890). L.B. acknowledges support from FONDAP Center for Astrophysics 15010003. We are grateful to the staff of IRAM-Granada for the help provided during the observations at the 30-m telescope. We also thank our referee for his valuable comments and suggestions.

\section{References}

Arce, H. G., Shepherd, D., Gueth, F., et al. 2007, PPV, 245

Battersby, C., Bally, J., Jackson, J. M., et al. 2010, ApJ, 721, 222

Beuther, H., \& Shepherd, D. 2005, CCSF, 105

Beuther, H., \& Sridharan, T. K. 2007, ApJ, 668, 348

Beuther, H., Schilke, P., Menten, K. M., et al. 2002, ApJ, 566, 945

Carey, S. J., Noriega-Crespo, A., Price, S., et al. 2005, BAAS, 37, 1252

Carey, S. J., Noriega-Crespo, A., Mizuno, D. R., et al. 2009, PASP, 121, 76

Cesaroni, R., Galli, D., Lodato, G., Walmsley, C. M., \& Zhang, Q. 2007, in Protostars and Planets V, 197 (Tucson: Univ. Arizona Press)

Codella, C., Cabrit, S., Gueth, F., et al. 2007, A\&A, 462, L53

Faúndez, S., Bronfman, L., Garay, G., et al. 2004, A\&A, 426, 97

Gibb, A. G., Richer, J. S., Chandler, C. J., \& Davis, C. J. 2004, ApJ, 603, 198

Gibb, A. G., Davis, C. J., \& Moore, T. J. T. 2007, MNRAS, 382, 1213

Giveon, U., Becker, R. H., Helfand, D. J., \& White, R. L. 2005, ApJ, 129, 348

Gueth, F., \& Guilloteau, S. 1999, A\&A, 343, 571

Guillet, V., Jones, A. P., Pineau Des Forêts, G. 2009, A\&A, 497, 145

Gusdorf, A., Cabrit, S., Flower, D. R., et al. 2008, A\&A, 482, 809

Hill, T., Burton, M. G., Minier, V., et al. 2005, MNRAS, 363, 405

Hofner, P., \& Churchwell, E. 1996, A\&AS, 120, 283

Jiménez-Serra, I., Caselli, P., Tan, J. C., et al. 2010, MNRAS, 406, 187

Krumholz, M. R., McKee, C. F., \& Klein, R. I. 2005, ApJ, 618, L33

López-Sepulcre, C. R., \& Walmsley, M. 2010, A\&A, 517, A66 (LS10)

McKee, C. F., \& Tan, J. C. 2002, Nature, 416, 59

McKee, C. F., \& Tan, J. C. 2003, ApJ, 585, 850

Miettinen, O., Harju, J., Haikala, L. K., \& Pomrén, C. 2006, A\&A, 460, 721

Motte, F., Bontemps, S., Schilke, P., et al. 2007, A\&A, 476, 1243

Rathborne, J. M., Jackson, J. M., \& Simon, R. 2006, ApJ, 641, 389

Rathborne, J. M., Jackson, J. M., Chambers, E. T. et al. 2010, ApJ, 715, 310

Ray, T., Dougados, C., Bacciotti, F., et al. 2007, PPV, 231

Sakai, T., Sakai, N., Hirota, T., \& Yamamoto, S. 2010, ApJ, 714, 1658

Schöier, F. L., van der Tak FFS., van Dishoeck, E. F., \& Black, J. H. 2005, A\&A, 432, 369

Schuller, F., Menten, K. M., Contreras, Y., et al. 2009, A\&A, 504, 415

Van der Tak, F. F. S., Black, J. H., Schöier, F. L., Jansen, D. J., et al. 2007, A\&A, 468, 627

Walsh, A. J., Burton, M. G., Hyland, A. R., et al. 1998, MNRAS, 301, 640

Wood, D. O. S., \& Churchwell, E. 1989, ApJ, 69, 831

Pages 5 to 13 are available in the electronic edition of the journal at http://www . aanda.org 
A. López-Sepulcre et al.: $\mathrm{SiO}$ outflows in high-mass star forming regions: A potential chemical clock?

\section{Appendix A: Tables}

Table A.1. Properties of the clumps derived from modified black body fits to their SEDs.

\begin{tabular}{|c|c|c|c|}
\hline Source & $\begin{array}{c}T_{\text {dust }} \\
(\mathrm{K})\end{array}$ & $\begin{array}{c}M \\
\left(M_{\odot}\right)\end{array}$ & $\begin{array}{l}L_{\text {bol }} \\
\left(L_{\odot}\right)\end{array}$ \\
\hline $05358+3543$ & 76 & 70 & 4370 \\
\hline G213.61-12.6* & 39 & 800 & 113000 \\
\hline $\mathrm{G} 189.78+0.34^{*}$ & 42 & 63 & 8630 \\
\hline G192.60-0.05* & 72 & 100 & 60200 \\
\hline 18151-1208_1* & 75 & 120 & 11800 \\
\hline G18.15-0.28 & 105 & 43 & 33400 \\
\hline G18.18-0.30 & 32 & 55 & 2640 \\
\hline $18223-1243$ & 73 & 230 & 16100 \\
\hline $18228-1312$ & 95 & 240 & 29800 \\
\hline $\mathrm{G} 19.27+0.1 \mathrm{M} 2$ & 29 & 57 & 1450 \\
\hline G19.27+0.1M1 & 33 & 47 & 2760 \\
\hline 18232-1154 & 53 & 61 & 19800 \\
\hline 18236-1205 & 60 & 320 & 3370 \\
\hline G19.61-0.24A* & 59 & 765 & 264000 \\
\hline G20.08-0.1 & 66 & 410 & 19700 \\
\hline 18264-1152 & 59 & 440 & 5970 \\
\hline $\mathrm{G} 23.60+0.0 \mathrm{M} 1$ & 21.5 & 660 & 3370 \\
\hline 18316-0602 & 48 & 610 & 171000 \\
\hline $\mathrm{G} 23.60+0.0 \mathrm{M} 2$ & 26 & 58 & 1780 \\
\hline 18317-0513 & 79 & 270 & 16200 \\
\hline $\mathrm{G} 24.08+0.0 \mathrm{M} 2 *$ & 31 & 93 & 2930 \\
\hline G24.33+0.1M1 & 56 & 460 & 17600 \\
\hline $\mathrm{G} 24.33+0.1 \mathrm{M} 4 *$ & 41 & 460 & 4890 \\
\hline $\mathrm{G} 24.33+0.1 \mathrm{M} 2 *$ & 28 & 400 & 7190 \\
\hline G24.60+0.1M2 & 21 & 390 & 2420 \\
\hline G24.60+0.1M1 & 22 & 140 & 772 \\
\hline G25.04-0.2M1 & 20 & 350 & 1320 \\
\hline G25.04-0.2M4* & 33 & 210 & 869 \\
\hline G25.04-0.2M2* & 25 & 100 & 1060 \\
\hline G28.28-0.35 & 109 & 97 & 45100 \\
\hline $\mathrm{G} 34.43+0.2 \mathrm{M} 1$ & 42 & 380 & 32700 \\
\hline $18507+0121$ & 61 & 820 & 17700 \\
\hline $\mathrm{G} 34.43+0.2 \mathrm{M} 3$ & 35 & 95 & 6940 \\
\hline G34.24+0.13 & 48 & 51 & 31700 \\
\hline $18517+0437^{*}$ & 43 & 400 & 27600 \\
\hline $19035+0641^{*}$ & 74 & 46 & 5830 \\
\hline $19095+0930$ & 61 & 260 & 52100 \\
\hline G43.89-0.38* & 57 & 88 & 53100 \\
\hline G61.48+0.09A* & 66 & 189 & 117000 \\
\hline $20216+4107^{*}$ & 72 & 17 & 3180 \\
\hline $20332+4124$ & 79 & 180 & 23900 \\
\hline $22134+5834^{*}$ & 82 & 55 & 12600 \\
\hline 22570+5912_1* & 82 & 180 & 48400 \\
\hline 23033+5951 & $71^{*}$ & 170 & 12500 \\
\hline NGC7538B* & 37 & 6480 & 200000 \\
\hline $23139+5939^{*}$ & 71 & 200 & 24100 \\
\hline $23151+5912^{*}$ & 98 & 180 & 121000 \\
\hline
\end{tabular}

Notes. ${ }^{*}$ Sources whose derived luminosities are upper limits (see Sect. 5.1). 
A\&A 526, L2 (2011)

Table A.2. Parameters of the $\mathrm{SiO}(2-1)$ and (3-2) lines, and $\mathrm{HCO}^{+}$outflow detection.

\begin{tabular}{|c|c|c|c|c|c|c|c|c|c|}
\hline \multirow[t]{2}{*}{ Sub-class } & \multirow[t]{2}{*}{ Source } & \multirow{2}{*}{$\begin{array}{c}\text { RA } \\
(\mathrm{J} 2000.0)\end{array}$} & \multirow{2}{*}{$\begin{array}{c}\text { Dec } \\
(\mathrm{J} 2000.0)\end{array}$} & \multirow{2}{*}{$\begin{array}{c}V_{\mathrm{LSR}^{*}} \\
\left(\mathrm{~km} \mathrm{~s}^{-1}\right)\end{array}$} & \multicolumn{2}{|c|}{$\mathrm{SiO}(2-1)$} & \multicolumn{2}{|c|}{$\mathrm{SiO}(3-2)$} & \multirow{2}{*}{$\begin{array}{c}\mathrm{HCO}^{+} \\
\text {outflow? }^{\ddagger} \\
(\mathrm{Y} / \mathrm{N})\end{array}$} \\
\hline & & & & & $\begin{array}{l}F W Z P^{\dagger} \\
\left(\mathrm{km} \mathrm{s}^{-1}\right)\end{array}$ & $\begin{array}{c}\int_{\left(\mathrm{K} \mathrm{km} \mathrm{s}^{-1}\right)} F_{\mathrm{v}} \mathrm{d} v^{\dagger} \\
.\end{array}$ & $\begin{array}{l}F W Z P^{\dagger} \\
\left(\mathrm{km} \mathrm{s}^{-1}\right)\end{array}$ & $\begin{array}{c}\int_{\left(\mathrm{K} \mathrm{km} \mathrm{s}^{-1}\right)} F_{\mathrm{v}} \mathrm{d} v^{\dagger} \\
x^{2}\end{array}$ & \\
\hline IRL & $05358+3543$ & $05: 39: 12.20$ & $+35: 45: 52.0$ & -15.8 & 38.3 & 6.5 & 50.2 & 6.8 & $\mathrm{Y}$ \\
\hline IRL & $\mathrm{G} 213.61-12.6$ & $06: 07: 49.23$ & $-06: 22: 40.6$ & 10.6 & 9.2 & 0.3 & 5.8 & 0.3 & $\mathrm{Y}$ \\
\hline IRL & $\mathrm{G} 189.78+0.34$ & $06: 08: 35.35$ & $+20: 39: 04.3$ & 9.2 & 15.2 & 1.1 & 21.0 & 2.0 & $\mathrm{Y}$ \\
\hline IRL & G192.58-0.04 & $06: 12: 52.90$ & $+18: 00: 35.0$ & 9.1 & 35.9 & 1.7 & 17.0 & 1.4 & Y \\
\hline IRL & G192.60-0.05 & $06: 12: 54.00$ & $+17: 59: 23.0$ & 7.4 & 29.2 & 1.6 & 23.1 & 2.6 & Y \\
\hline IRD & 18151-1208_2 & $18: 17: 50.50$ & $-12: 07: 55.0$ & 29.8 & 84.3 & 5.7 & 103.1 & 11.6 & Y \\
\hline IRL & 18151-1208_3 & $18: 17: 52.30$ & $-12: 06: 56.0$ & 30.7 & - & - & - & - & $\mathrm{N}$ \\
\hline IRL & 18151-1208_1 & $18: 17: 58.00$ & $-12: 07: 27.0$ & 33.3 & 36.3 & 1.2 & 31.1 & 1.7 & $\mathrm{Y}$ \\
\hline IRL & G18.15-0.28 & $18: 25: 02.41$ & $-13: 15: 25.7$ & 55.8 & - & - & - & - & $\mathrm{N}$ \\
\hline IRD & G18.18-0.30 & $18: 25: 07.30$ & $-13: 14: 23.0$ & 50.0 & 35.9 & 1.8 & 29.8 & 1.4 & Y \\
\hline IRL & $18223-1243$ & $18: 25: 10.87$ & $-12: 42: 26.8$ & 45.2 & 21.2 & 0.4 & 13.0 & 0.3 & $\mathrm{Y}$ \\
\hline IRL & $18228-1312$ & $18: 25: 42.35$ & $-13: 10: 18.1$ & 33.1 & 15.2 & 0.4 & 27.9 & 0.4 & $\mathrm{Y}$ \\
\hline IRD & $\mathrm{G} 19.27+0.1 \mathrm{M} 2$ & $18: 25: 52.60$ & $-12: 04: 48.0$ & 26.9 & 66.3 & 4.0 & 51.0 & 3.7 & $\mathrm{Y}$ \\
\hline IRD & $\mathrm{G} 19.27+0.1 \mathrm{M} 1$ & $18: 25: 58.50$ & $-12: 03: 59.0$ & 26.5 & 75.9 & 6.2 & 69.9 & 9.1 & $\mathrm{Y}$ \\
\hline IRL & $18232-1154$ & $18: 26: 04.37$ & $-11: 52: 33: 4$ & 24.4 & - & - & - & - & - \\
\hline IRL & $18236-1205$ & $18: 26: 25.41$ & $-12: 03: 51.4$ & 26.5 & 51.1 & 3.0 & 34.8 & 2.4 & $\mathrm{Y}$ \\
\hline IRL & G19.61-0.24A & $18: 27: 38: 16$ & $-11: 56: 40.2$ & 42.4 & 51.1 & 7.8 & 59.0 & 17.0 & - \\
\hline IRL & G20.08-0.14 & $18: 28: 10: 28$ & $-11: 28: 48.7$ & 42.5 & 41.9 & 8.7 & 50.0 & 16.2 & - \\
\hline IRL & $18264-1152$ & $18: 29: 14.40$ & $-11: 50: 21.3$ & 43.9 & 71.1 & 6.2 & 40.1 & 6.2 & $\mathrm{Y}$ \\
\hline IRD & $\mathrm{G} 23.60+0.0 \mathrm{M} 1$ & $18: 34: 11.60$ & $-08: 19: 06.0$ & 106.5 & 73.1 & 5.0 & 82.9 & 6.1 & $\mathrm{Y}$ \\
\hline IRL & $18316-0602$ & $18: 34: 20.46$ & $-05: 59: 30.4$ & 42.5 & 75.1 & 8.5 & 60.3 & 9.3 & $\mathrm{Y}$ \\
\hline IRD & $\mathrm{G} 23.60+0.0 \mathrm{M} 2$ & $18: 34: 21.10$ & $-08: 18: 07.0$ & 53.6 & 85.1 & 5.6 & 57.9 & 4.5 & $\mathrm{Y}$ \\
\hline IRL & $18317-0513$ & $18: 34: 25.94$ & $-05: 10: 48.6$ & 42.0 & 16.4 & 0.4 & 9.8 & 0.3 & $\mathrm{~N}$ \\
\hline IRD & $\mathrm{G} 24.08+0.0 \mathrm{M} 2$ & $18: 34: 51.10$ & $-07: 45: 32.0$ & 52.0 & 21.2 & 0.9 & 17.0 & 0.4 & $\mathrm{Y}$ \\
\hline IRD & $\mathrm{G} 24.33+0.1 \mathrm{M} 1$ & $18: 35: 07.90$ & $-07: 35: 04.0$ & 113.6 & 49.1 & 4.8 & 55.0 & 5.4 & $\mathrm{Y}$ \\
\hline IRD & $\mathrm{G} 24.33+0.1 \mathrm{M} 4$ & $18: 35: 18.56$ & $-07: 37: 26.2$ & 114.3 & 8.4 & 0.3 & - & - & $\mathrm{N}$ \\
\hline IRD & $\mathrm{G} 24.33+0.1 \mathrm{M} 2$ & $18: 35: 34.50$ & $-07: 37: 28.0$ & 118.6 & 33.1 & 1.6 & 44.6 & 1.7 & $\mathrm{~N}$ \\
\hline IRD & $\mathrm{G} 24.60+0.1 \mathrm{M} 2$ & $18: 35: 35.70$ & $-07: 18: 09.0$ & 115.3 & 53.1 & 2.1 & 43.0 & 2.4 & $\mathrm{Y}$ \\
\hline IRD & $\mathrm{G} 24.60+0.1 \mathrm{M} 1$ & $18: 35: 40.20$ & $-07: 18: 37.0$ & 53.2 & 49.9 & 3.8 & 67.0 & 4.2 & $\mathrm{Y}$ \\
\hline IRD & G25.04-0.2M1 & $18: 38: 10.20$ & $-07: 02: 34.0$ & 63.8 & 66.3 & 4.0 & 52.1 & 3.4 & $\mathrm{Y}$ \\
\hline IRD & G25.04-0.2M4 & $18: 38: 13.70$ & $-07: 03: 12.0$ & 63.8 & 18.4 & 0.7 & 13.0 & 0.5 & $\mathrm{Y}$ \\
\hline IRD & $\mathrm{G} 25.04-0.2 \mathrm{M} 2$ & $18: 38: 17.70$ & $-07: 02: 51.0$ & 63.9 & 30.0 & 2.3 & 24.2 & 1.7 & $\mathrm{Y}$ \\
\hline IRL & G28.28-0.35 & $18: 44: 14.20$ & $-04: 17: 59.0$ & 48.7 & 8.4 & 0.3 & - & - & $\mathrm{N}$ \\
\hline IRD & $\mathrm{G} 34.43+0.2 \mathrm{M} 1$ & $18: 53: 18.00$ & $+01: 25: 23.0$ & 58.1 & 75.1 & 14.4 & 63.0 & 18.2 & $\mathrm{Y}$ \\
\hline IRL & $\mathrm{G} 34.26+0.15$ & $18: 53: 18.44$ & $+01: 14: 57.8$ & 58.4 & 25.2 & 6.6 & 24.2 & 13.4 & - \\
\hline IRL & $18507+0121$ & $18: 53: 19.58$ & $+01: 24: 37.1$ & 58.2 & 57.1 & 7.8 & 52.1 & 6.4 & $\mathrm{Y}$ \\
\hline IRD & $\mathrm{G} 34.43+0.2 \mathrm{M} 3$ & $18: 53: 20.40$ & $+01: 28: 23.0$ & 59.4 & 85.9 & 12.9 & 72.0 & 13.9 & Y \\
\hline IRL & G34.24+0.13 & $18: 53: 21.70$ & $+01: 13: 37.0$ & 57.2 & 18.0 & 0.9 & 34.0 & 1.5 & $\mathrm{~N}$ \\
\hline IRL & $18517+0437$ & $18: 54: 14.30$ & $+04: 41: 40.0$ & 44.1 & 13.2 & 2.4 & 26.0 & 3.8 & Y \\
\hline IRD & $\mathrm{G} 34.77-0.6 \mathrm{M} 2$ & $18: 56: 49.41$ & $+01: 23: 16.0$ & 42.2 & - & - & - & - & $\mathrm{N}$ \\
\hline IRD & G35.39-0.3M4 & 18:57:07.90 & $+02: 08: 23.0$ & 44.6 & 15.2 & 0.5 & 9.0 & 0.5 & $\mathrm{~N}$ \\
\hline IRL & G35.20-1.74 & 19:01:46.40 & $+01: 13: 24.5$ & 45.0 & 33.1 & 0.7 & 32.2 & 1.2 & - \\
\hline IRD & G38.95-0.5M1 & 19:04:07.40 & $+05: 08: 48.0$ & 42.3 & 32.4 & 1.8 & 14.9 & 1.1 & $\mathrm{Y}$ \\
\hline IRL & $19035+0641$ & 19:06:01.60 & $+06: 46: 43.0$ & 32.8 & 24.4 & 1.5 & 30.0 & 1.4 & $\mathrm{Y}$ \\
\hline IRL & $19095+0930$ & $19: 11: 54.02$ & $+09: 35: 52.0$ & 43.9 & 42.3 & 4.8 & 55.8 & 7.1 & $\mathrm{Y}$ \\
\hline IRL & G43.89-0.38 & $19: 14: 26.07$ & $+09: 22: 33.9$ & 53.7 & 41.9 & 1.4 & 47.8 & 7.1 & - \\
\hline IRL & $\mathrm{G} 61.48+0.09 \mathrm{~A}$ & $19: 46: 49.11$ & $+25: 12: 48.0$ & 21.5 & 11.2 & 0.5 & 12.0 & 0.5 & - \\
\hline IRL & G75.78-0.34 & $20: 21: 44.01$ & $+37: 26: 39.4$ & 0.3 & 36.3 & 4.4 & 40.9 & 6.9 & - \\
\hline IRL & $20216+4107$ & $20: 23: 23.60$ & $+41: 17: 38.0$ & -1.6 & - & - & - & - & $\mathrm{Y}$ \\
\hline IRL & $20332+4124$ & $20: 34: 59.90$ & $+41: 34: 49.0$ & -2.7 & - & - & - & - & $\mathrm{N}$ \\
\hline IRL & $22134+5834$ & $22: 15: 09.30$ & $+58: 49: 06.0$ & -18.3 & - & _- & - & _- & Y \\
\hline IRL & $22570+5912 \_2$ & $22: 58: 58.75$ & $+59: 27: 29.2$ & -47.8 & 12.0 & 0.5 & 22.1 & 0.7 & $\mathrm{~N}$ \\
\hline IRL & $22570+5912 \_1$ & 22:59:05.90 & $+59: 28: 19.0$ & -45.6 & - & - & 5.0 & 0.2 & $\mathrm{~N}$ \\
\hline IRL & $23033+5951$ & $23: 05: 25.50$ & $+60: 08: 06.0$ & -53.1 & 33.9 & 2.2 & 52.9 & 2.6 & $\mathrm{Y}$ \\
\hline IRL & NGC7538B & $23: 13: 45.25$ & $+61: 28: 10.2$ & -57.0 & 38.3 & 4.3 & 57.1 & 5.3 & - \\
\hline IRL & $23139+5939$ & $23: 16: 11.12$ & $+59: 55: 30.8$ & -44.1 & 61.1 & 4.4 & 59.0 & 6.1 & $\mathrm{Y}$ \\
\hline IRL & $23151+5912$ & $23: 17: 21.00$ & $+59: 28: 49.0$ & -54.5 & 25.2 & 0.8 & 27.1 & 1.3 & $\mathrm{Y}$ \\
\hline
\end{tabular}

Notes. ${ }^{(*)}$ Systemic velocity of the clump as measured from the peak velocity of the optically thin $\mathrm{H}^{13} \mathrm{CO}^{+}(1-0)$ line, observed simultaneously to $\mathrm{SiO}(2-1)$ and $\mathrm{SiO}(3-2) .{ }^{(\dagger)}$ Not detected. ${ }^{(\ddagger)}$ No observation made towards this source (see Sect. 2). 
A. López-Sepulcre et al.: SiO outflows in high-mass star forming regions: A potential chemical clock?

\section{Appendix B: Figures}

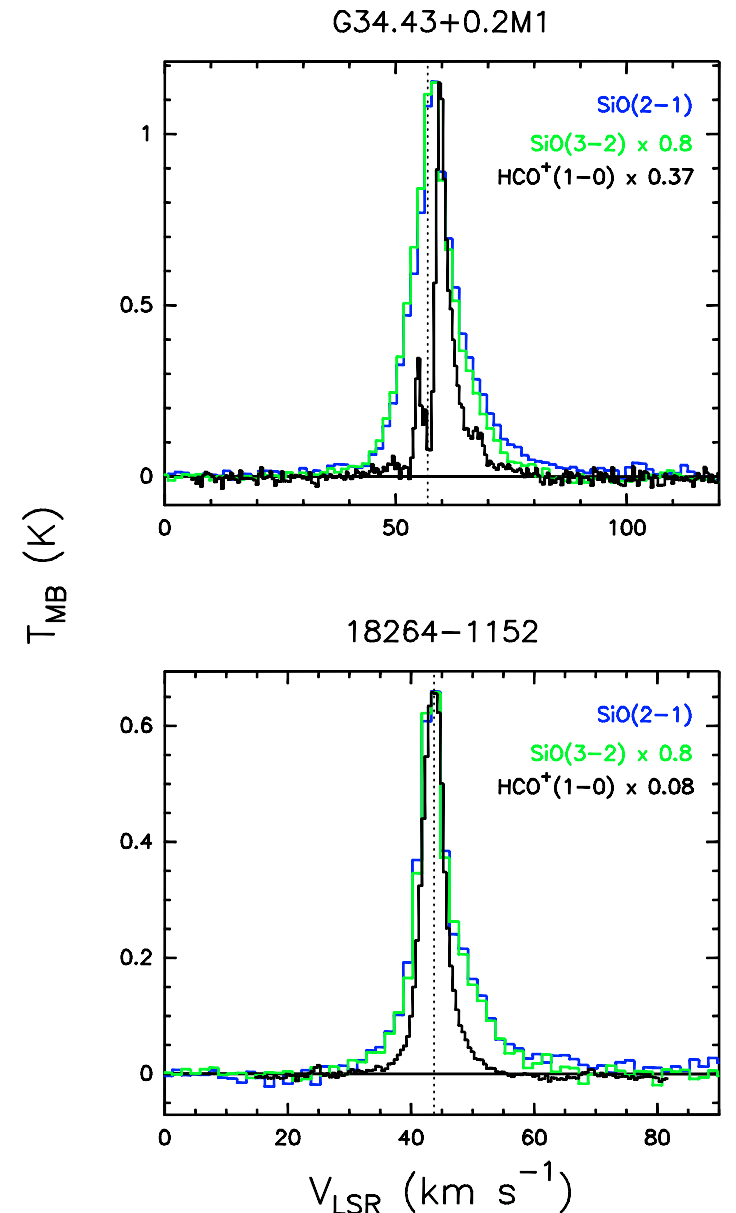

Fig. B.1. $\mathrm{SiO}(2-1)$ (blue), $\mathrm{SiO}(3-2)$ (green), and $\mathrm{HCO}^{+}(1-0)($ black) spectra observed towards the IR-dark source G34.43+0.2M1 and the IR-loud source 18264-1152. The vertical dotted lines mark the systemic velocity of the clump, listed in Col. 5 of Table A.2. 


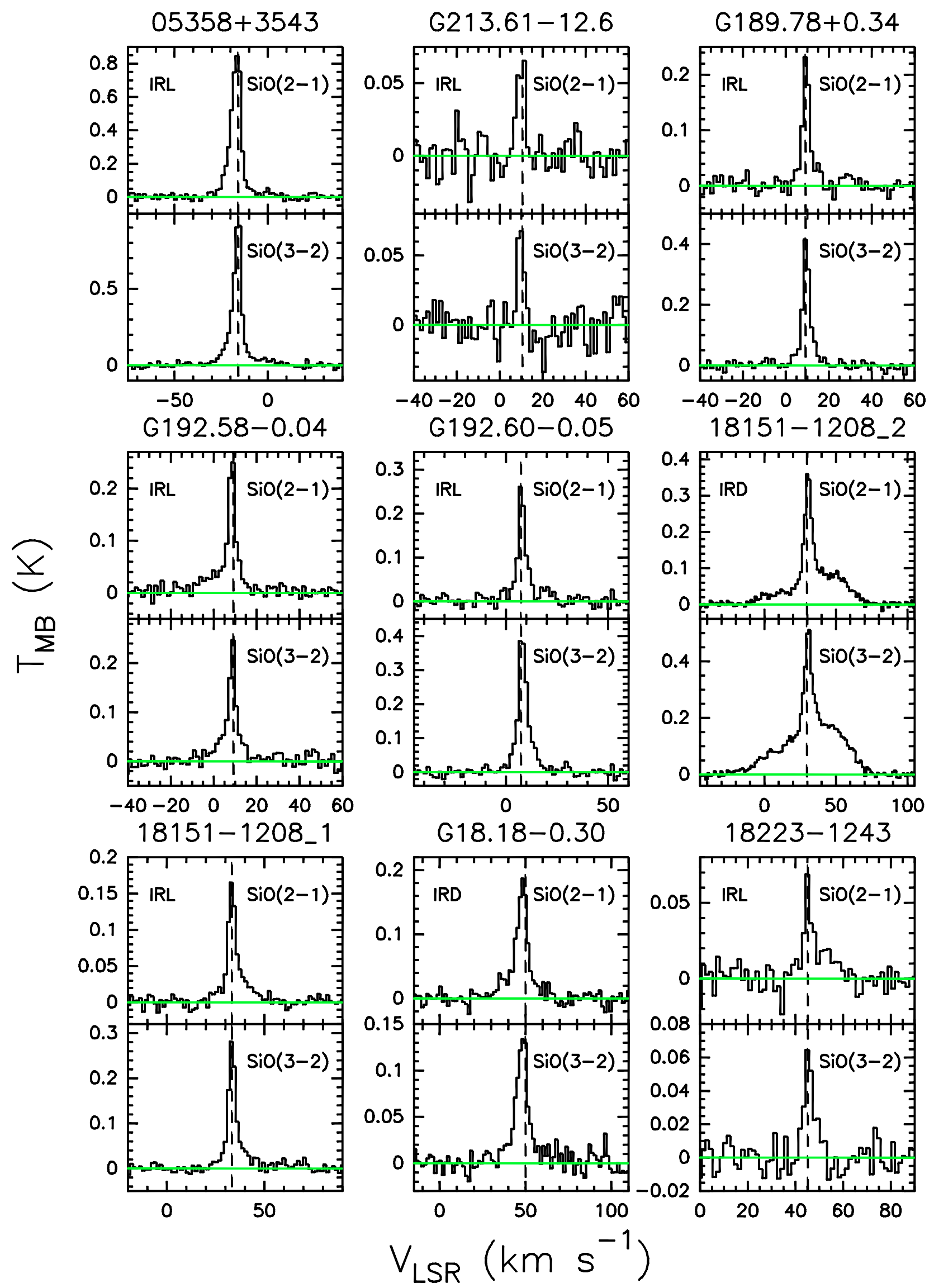

Fig. B.2. $\mathrm{SiO}(2-1)$ and (3-2) spectra. The dashed vertical line marks the systemic velocity of the clump, listed in Col. 5 of Table A.2. 
A. López-Sepulcre et al.: $\mathrm{SiO}$ outflows in high-mass star forming regions: A potential chemical clock?
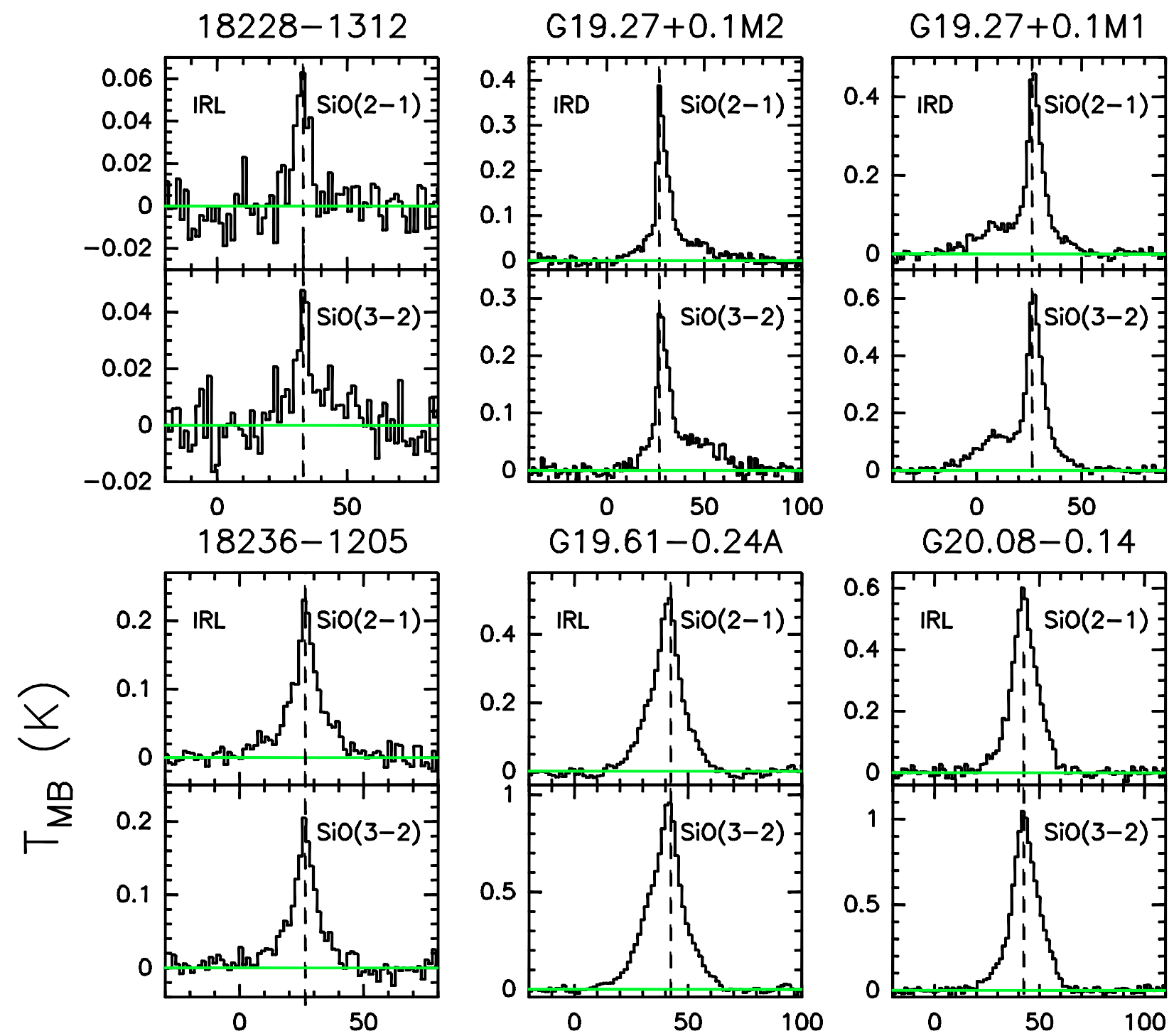

G20.08-0.14
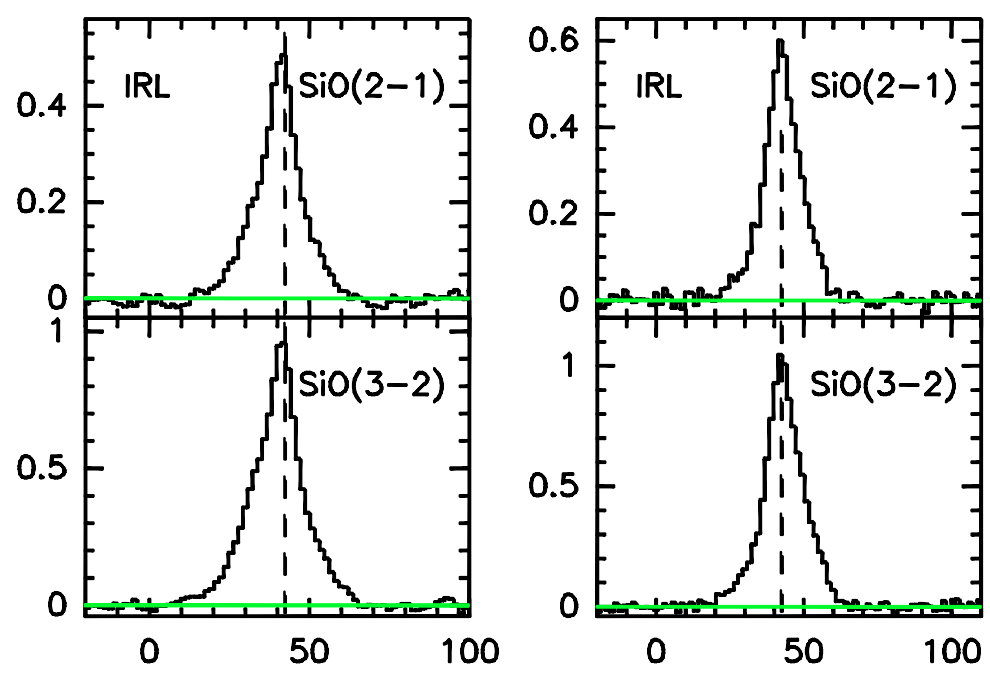

G23.60+0.0M1

18316-0602
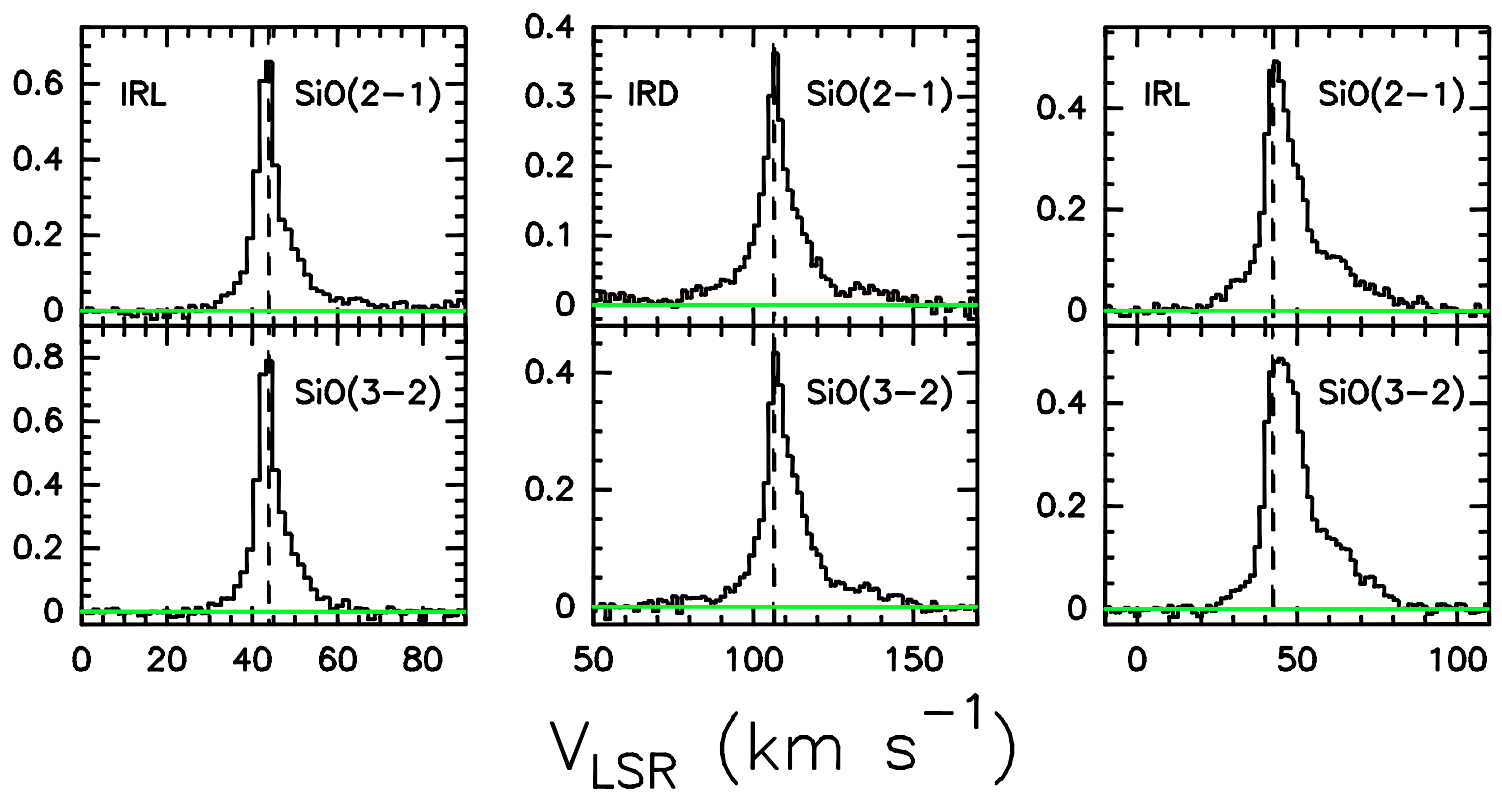

Fig. B.2. continued. 


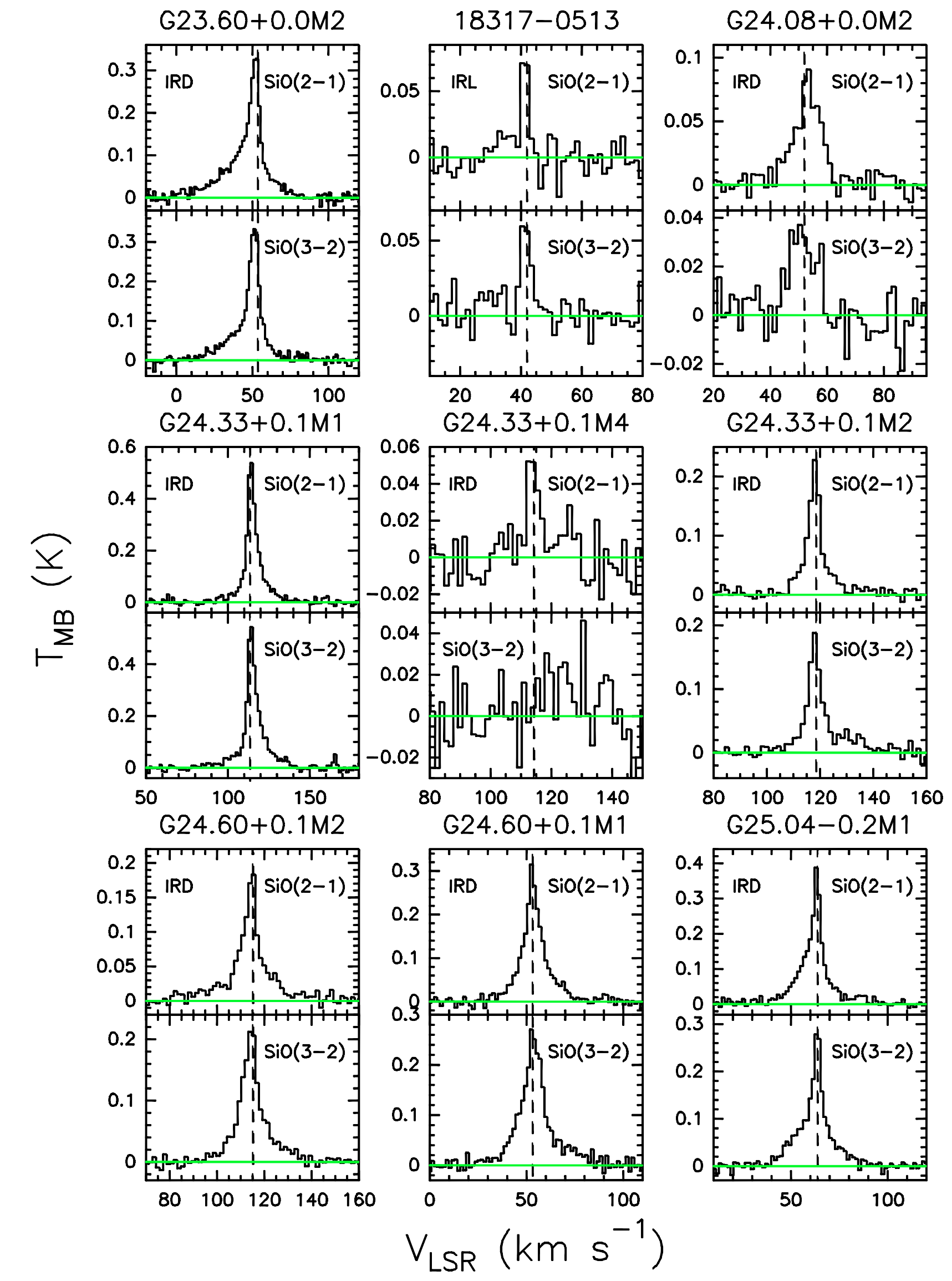

Fig. B.2. continued. 
A. López-Sepulcre et al.: SiO outflows in high-mass star forming regions: A potential chemical clock?
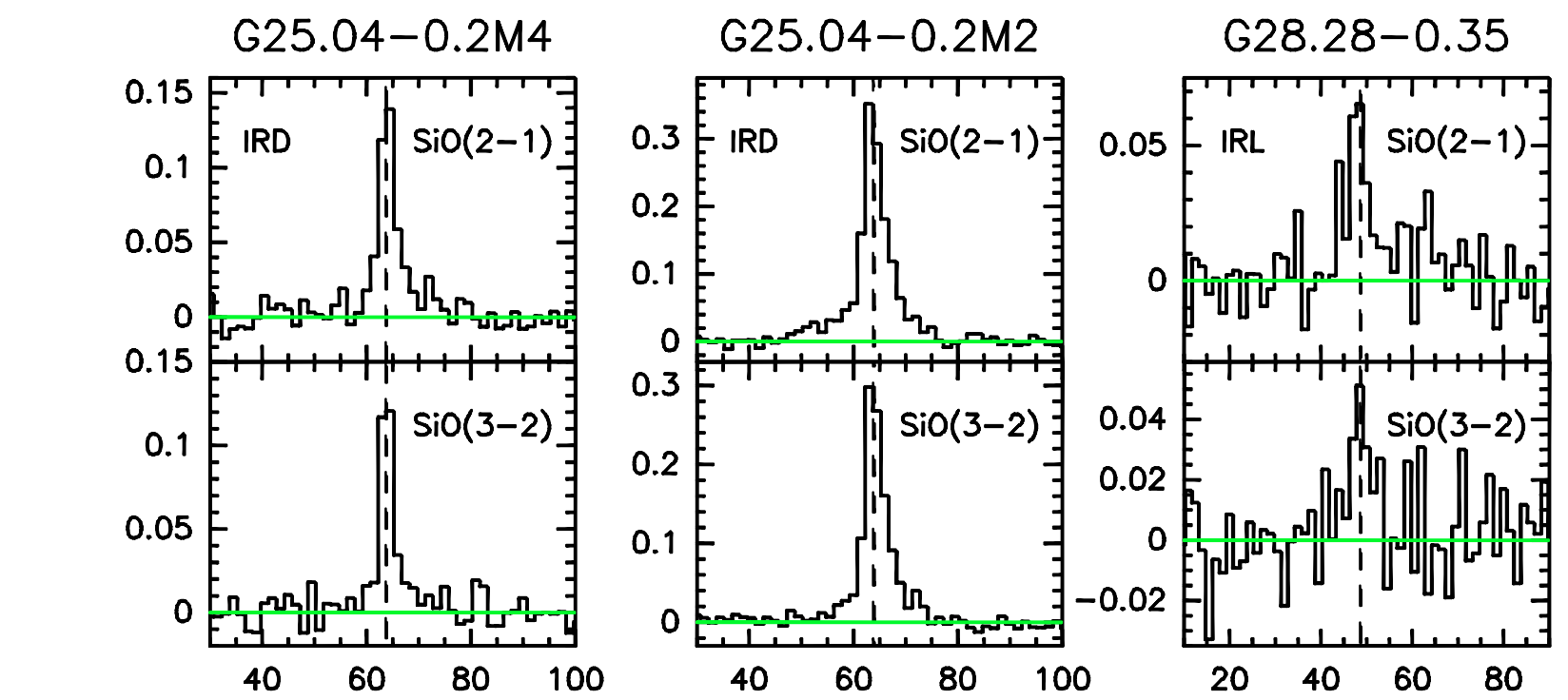

G34.43+0.2M 1

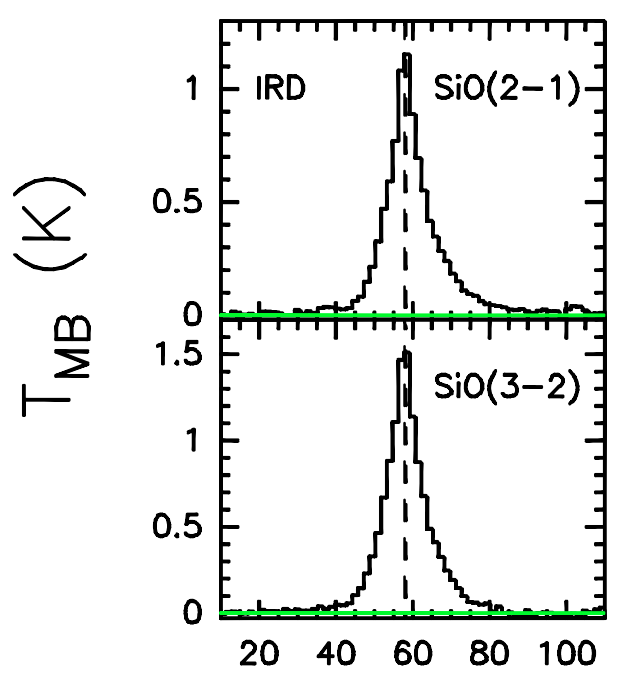

G34.26+0.15

$18507+0121$
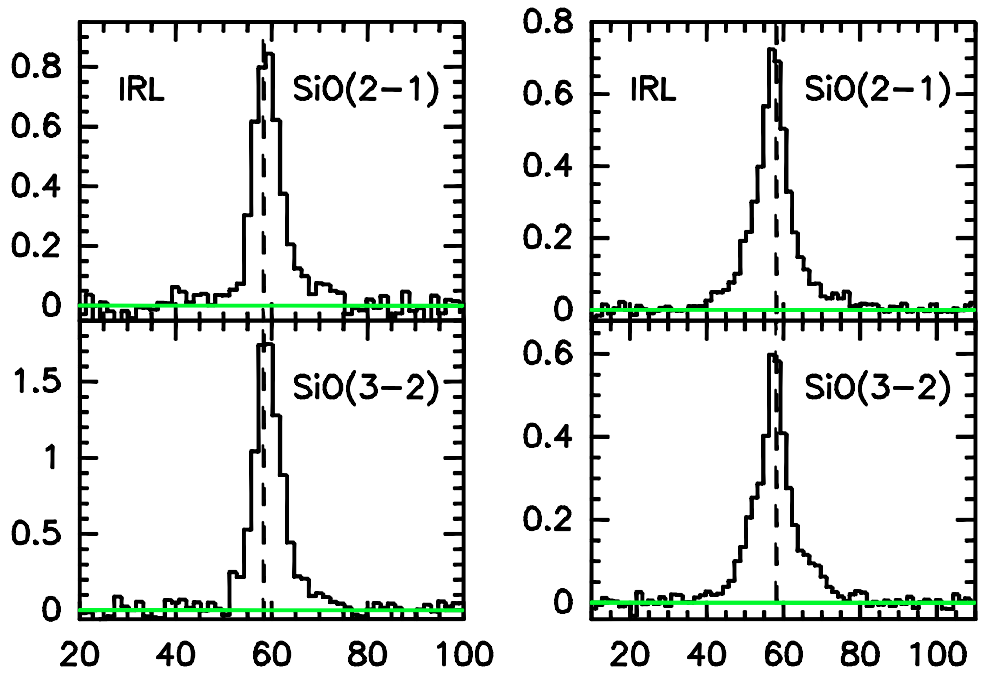

$\mathrm{G} 34.43+0.2 \mathrm{M} 3$
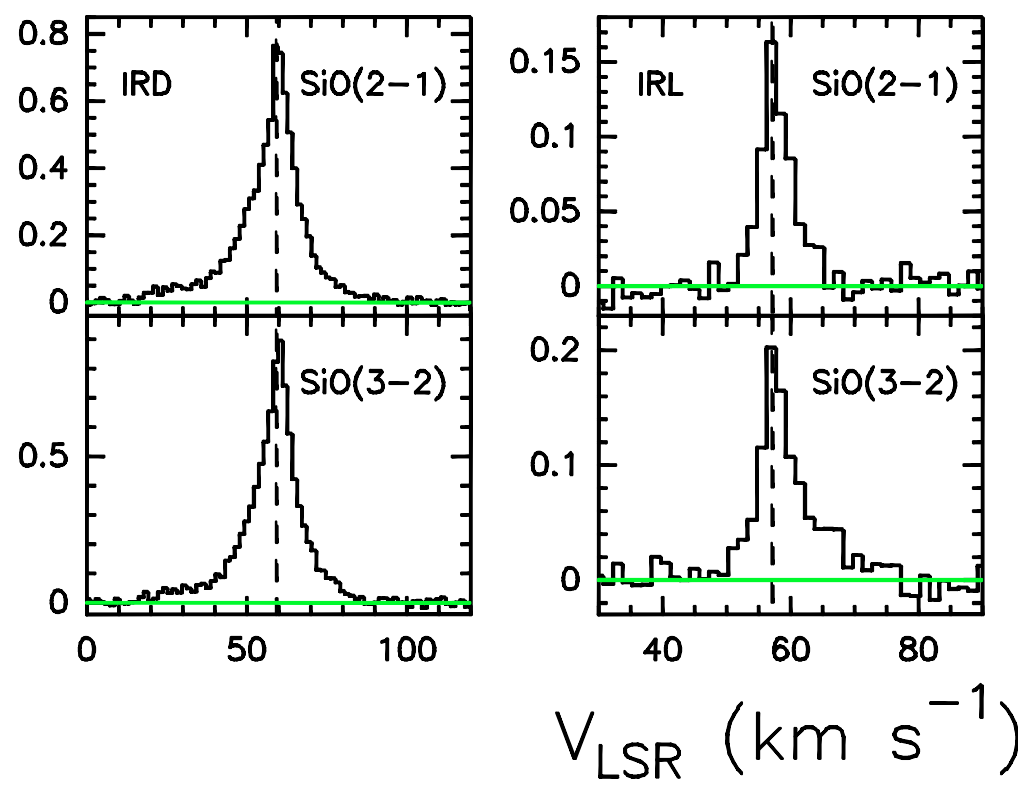

$18517+0437$

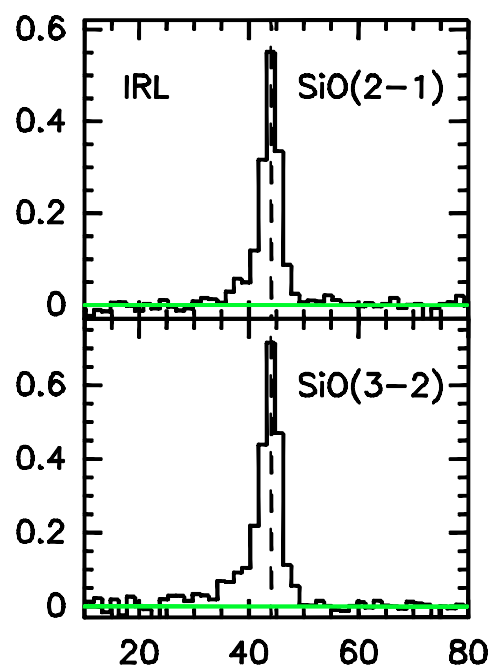

Fig. B.2. continued. 
G35.39-0.3M4
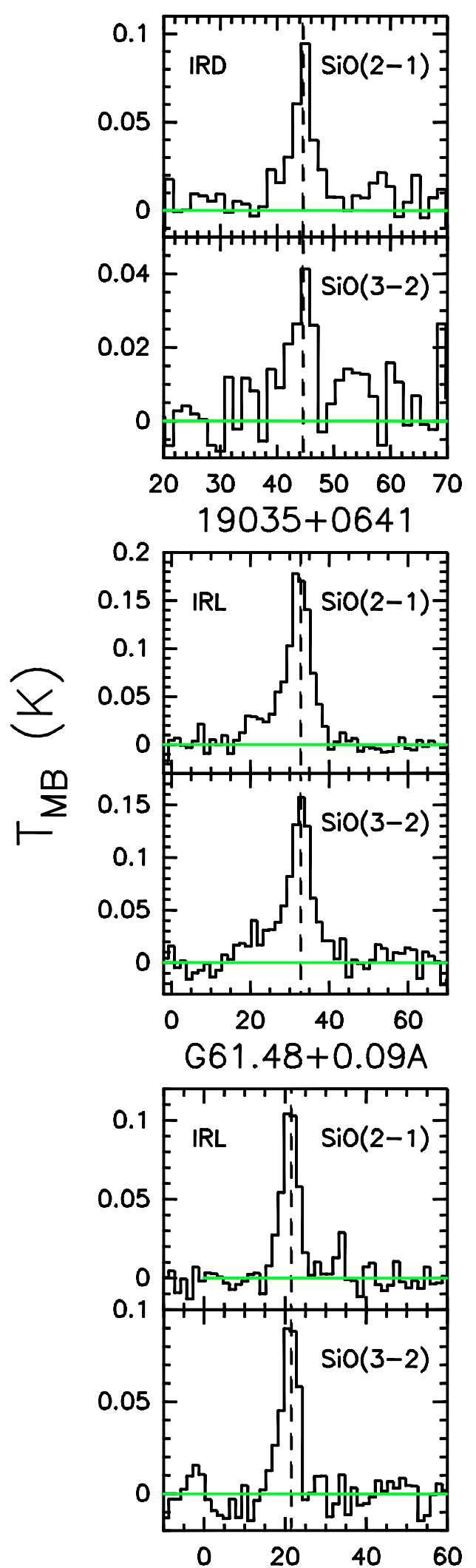

G35.20-1.74
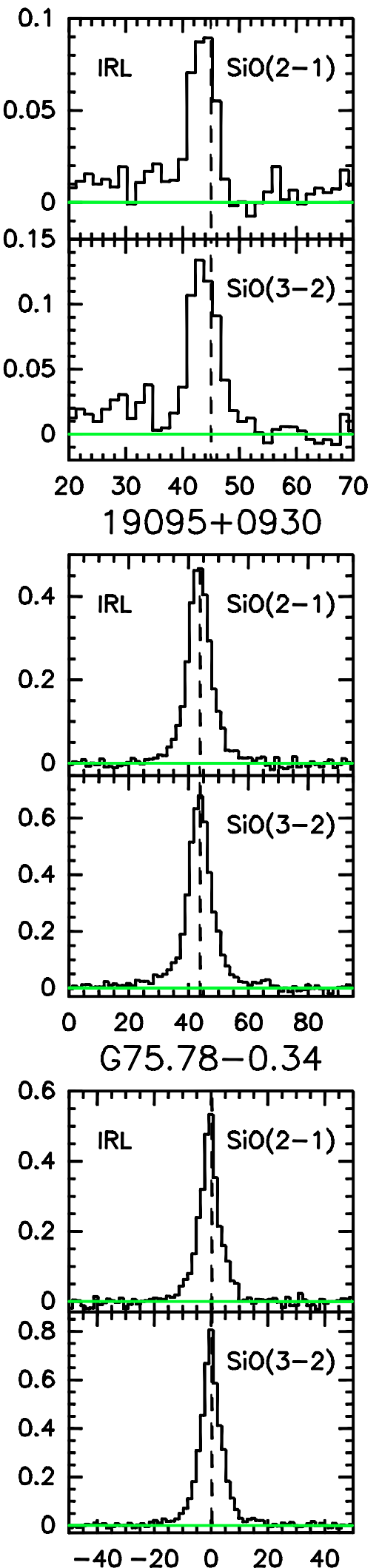

$V_{\mathrm{LSR}}\left(\mathrm{km} \mathrm{s}^{-1}\right)$
G38.95-0.5M1

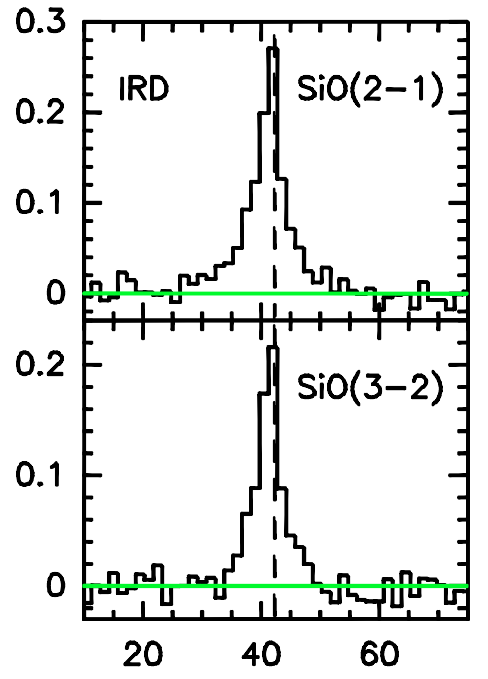

G43.89-0.38
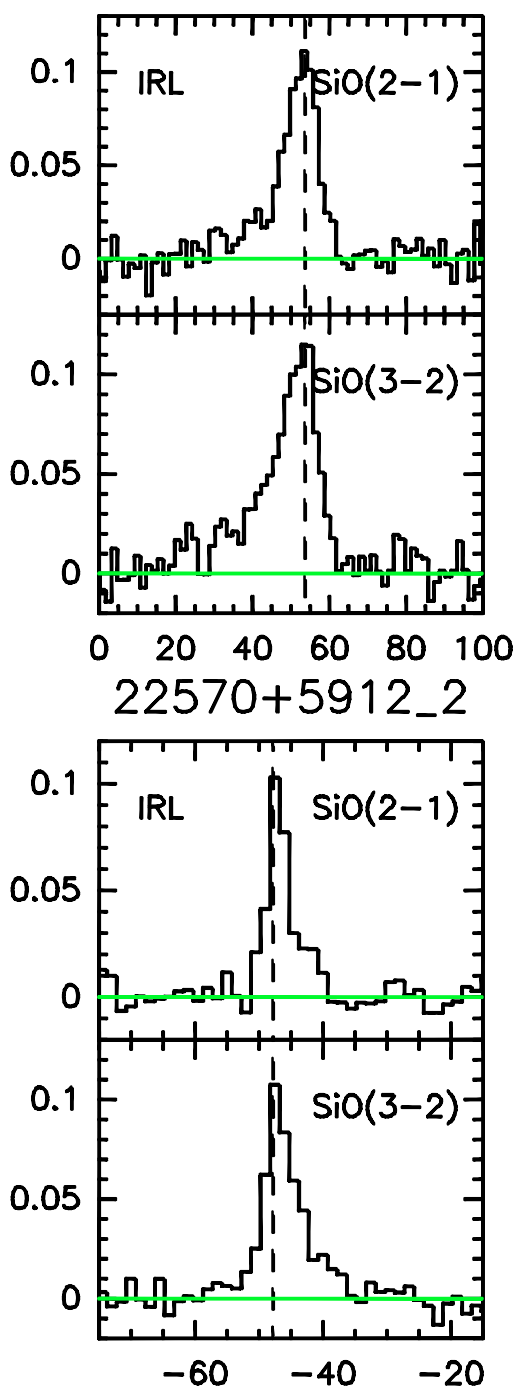

Fig. B.2. continued. 
A. López-Sepulcre et al.: $\mathrm{SiO}$ outflows in high-mass star forming regions: A potential chemical clock?

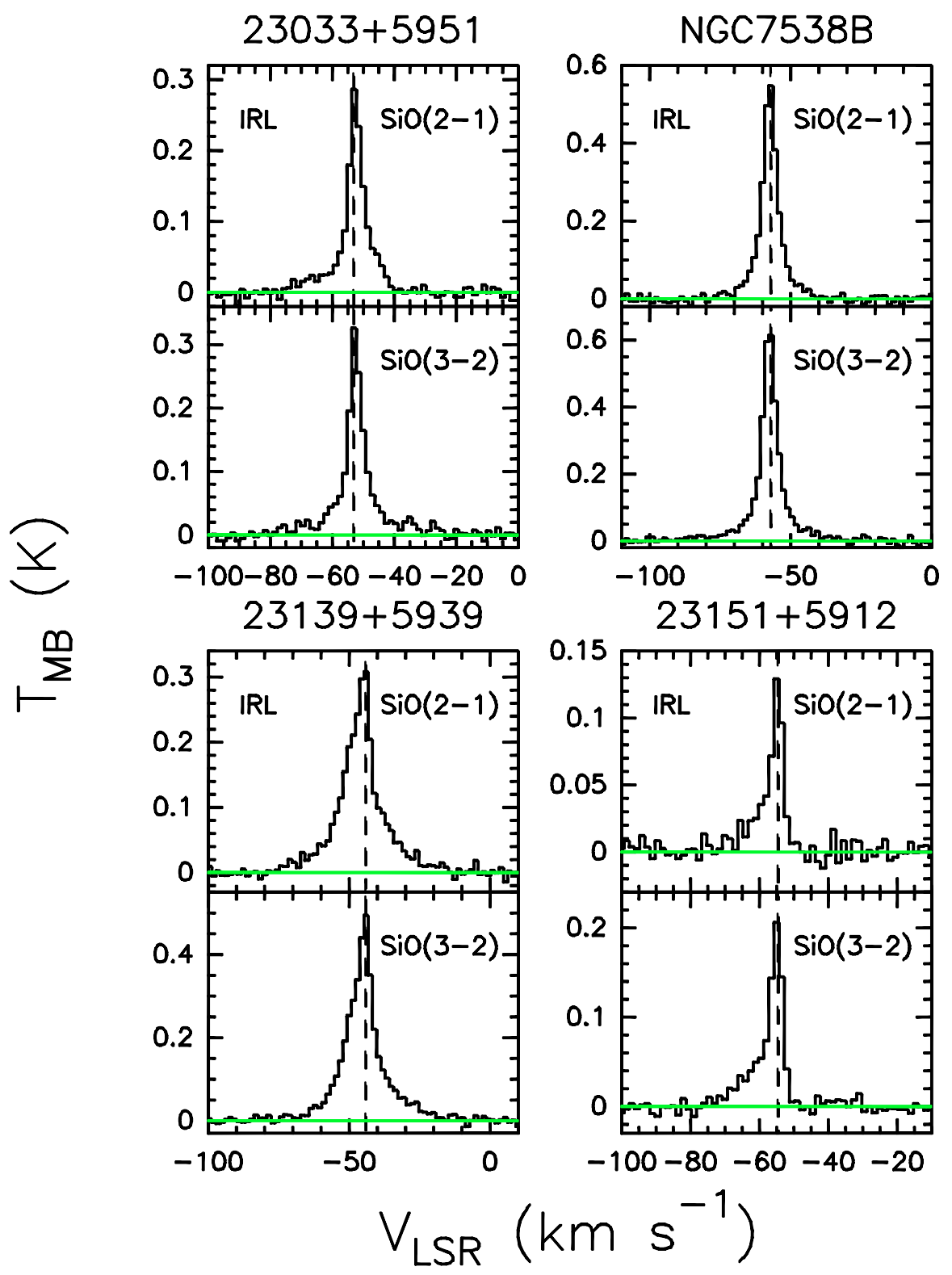

Fig. B.2. continued. 